\title{
Insular Type of Papillary Thyroid Carcinoma- A Case Report and Literature Review
}

\author{
Khadija Jamal Saad ${ }^{1,2,}$, Carine Ghassan Richa ${ }^{1,3}$, Randa Mohammad Chehab ${ }^{4}$, George Phillip \\ Aftimos $^{5}$, Elie Mekhael Gharios ${ }^{1,6}$ and Marie Tanios Merheb ${ }^{1,7}$ \\ ${ }^{1}$ Department of Endocrinology, Mount Lebanon Hospital, Beirut, Lebanon \\ ${ }^{2}$ Endocrinology fellow, Lebanese University, Hadath, Lebanon \\ ${ }^{3}$ Endocrinology fellow, Lebanese University, Hadath, Lebanon \\ ${ }^{4}$ Anathomo-pathology resident, Lebanese University, Hadath, Lebanon \\ ${ }^{5}$ Anathomo-pathologist, National institute of pathology, Lebanon \\ ${ }^{6}$ Clinical Endocrinology, Mount Lebanon Hospital, Beirut, Lebanon \\ ${ }^{7}$ Clinical Endocrinology, Mount Lebanon Hospital, Beirut, Lebanon
}

Received: April 19, 2018; Accepted: May 08, 2018; Published: May 18, 2018

*Corresponding author: Khadija Jamal Saad,Endocrinology fellow,Lebanese university, Lebanon, Tel: +961 70 281372; E-mail: Khadijasaad9@ gmail.com

\begin{abstract}
Introduction: Insular variant of papillary thyroid carcinoma is a rare pattern. Here, we report a case of poorly differentiated invasive insular variant of papillary thyroid cancer.

Case: A 55 year-old lady presented with 5 years history of an anterior neck mass. In fact she had thyroidectomy performed many years ago and she was on levothyroxine suppression therapy. Thyroid ultrasound revealed right thyroid heterogeneous macrocalcification with compression of the trachea. She was treated with surgery and histology confirmed "insular thyroid papillary carcinoma growth. She received multiple sessions of radiotherapy planned to receive radioactive iodine.

Conclusion: Insular thyroid carcinoma is a rare entity of papillary cancer. It is aggressive in nature and has a poor prognosis. This pathology requires an early diagnosis and treatment in order to improve the survival.
\end{abstract}

Keywords: Insular; papillary; thyroid cancer

\section{Introduction}

Insular thyroid carcinoma is a rare subtype of papillary carcinoma which is a part of thyroid follicular epithelial derived cancer.

It is mainly classified as aggressive subtype of well differentiated cancer or less aggressive form of poorly differentiated cancer. It occurs more commonly in females. Clinical features are usually non specific. It is mainly diagnosed by histopathology and staged according to International Staging System. This entity is usually differentiated from other forms of thyroid cancer by immunohistochemistry. Dedifferentiation can occur and it can progress to anaplastic cancer.
Total thyroidectomy is the gold standard of treatment.

Insular thyroid carcinoma has a high incidence of metastasis, recurrence, mortality and poor prognosis. This prompts early diagnosis and treatment. Here, we will discuss a case of insular thyroid carcinoma diagnosed by histology and treated with thyroidectomy followed by radiotherapy and planned for radioactive iodine.

\section{Case presentation}

A 55 year-old female, with no previous radiation exposure, presented with slow growing right anterior neck mass of 5 years duration. It has been increasing in size more rapidly in the last month.

She had right subtotal thyroidectomy 26 years ago for goiter and pathology result tend to be benign, and since then she has been started on levothyroxine replacement at a dose of 100 micrograms daily.

Physical examination revealed a right movable mass, hard in consistency, associated with hoarseness, dyspnea and stridor.

The thyroid ultrasound exam showed right thyroidal mass with calcification. The CT scan of the neck demonstrated a right thyroid heterogeneous macro calcification mass causing mass effect and displacement of the trachea. FNA done, it revealed huge asymmetrical nodular thyroid enlargement ,the aspirate reveal cellular cluster of malignant follicular cells with extremely low cytomorphological nuclear grade with papillary configuration ,obvious nuclear criteria consistent with malignant right papillary thyroid carcinoma, immunohistochemistry done thyroglobulin stain and CK 19 were positive.

Thyroid function tests revealed a thyroid stimulating 
hormone (TSH) level of 0.158 (0.35-4.94 UI/ml) on levothyroxine 100 micrograms daily and thyroglobulin level of $11700 \mathrm{ng} / \mathrm{ml}$ (normal range: 0.73-84). (Table 1)

Extrinsic compression of the trachea with severe left tracheal deviation was seen on chest imaging. (Figure 1)

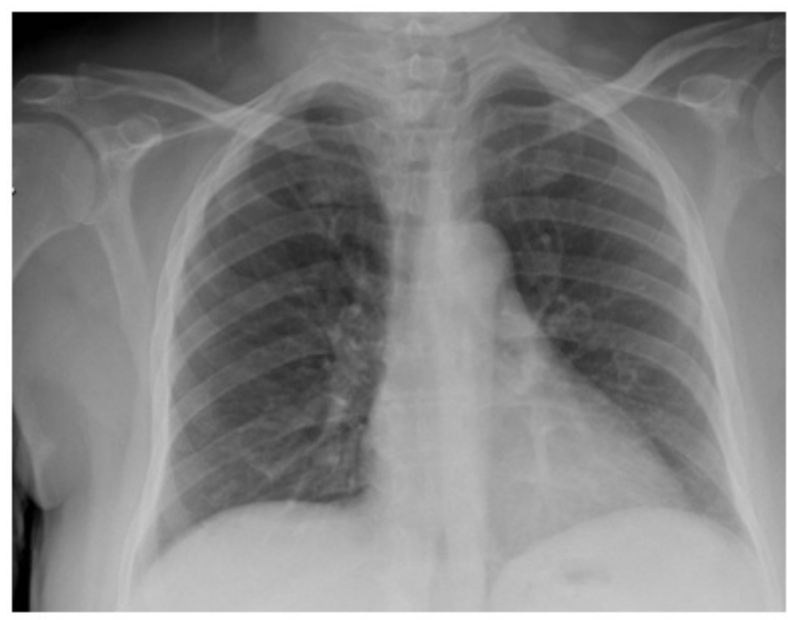

Figure 1: left tracheal deviation

\section{Table 1: initial laboratory workup}

\begin{tabular}{|l|l|}
\hline White blood cells(4500-10500 cell/microliter) & 14900 \\
\hline Creatinine $(0.6-1.2 \mathrm{mg} / \mathrm{dl})$ & 0.38 \\
\hline Hemoglobin $(12-15.5 \mathrm{~g} / \mathrm{dl})$ & 13 \\
\hline Potassium $(3.5-5.1 \mathrm{nmol} / \mathrm{l})$ & 2.3 \\
\hline Sodium (135-145 nmol /l) & 139 \\
\hline Direct bilirubin (<0.3 mg/dl) & 0.55 \\
\hline Total bilirubin( 0.1-1.2 mg/dl) & 0.84 \\
\hline Amylase (10-120 U/L) & 340 \\
\hline Lipase (13-60 U/L) & 820 \\
\hline Calcium (8.5-10.2 mg/dl) & 11 \\
\hline Albumin (36-50 g/l) & 38 \\
\hline Phosphorus (2.5-4.5 mg/dl) & 2.03 \\
\hline Magnesium (1.58-2.55 mg/dl) & 1.85 \\
\hline Thyroid stimulating hormone (0.35-4.94 Ul/ml) & 0.158 \\
\hline
\end{tabular}

As treatment patient underwent total thyroidectomy.

Intraoperative findings showed highly vascularized, huge tumor of the right lobe with extension to the left ,to the trachea and the esophagus, with difficult dissection from the other anatomic structure that is associated with severe adhesions.

Postoperatively, hypocalcemia of $7.9 \mathrm{mg} / \mathrm{dl}$ (normal range 8.5-10.2) and hypophosphatemia of $2.1 \mathrm{mg} / \mathrm{dl}$ (normal range 2.54.5) developed and our patient failed multiple trials of extubation. Finally, a tracheostomy was performed.
After correction of electrolyte disturbances, the patient leaved the hospital on levothyroxine 150 micrograms daily, awaiting the pathology report.

Anatomopathology performed it showed macroscopically, a thyroid fragment, weighing 114 grams with $6.5 \times 5.5 \times 2 \mathrm{~cm}$ in diameter was observed, and a tumor occupying the total parenchyma with a hemorrhagic foci measuring $6.8 \times 4.7 \times 4 \mathrm{~cm}$ was noticed.

Whereas, microscopically, the fragment showed poorly limited, infiltrating, proliferative tumor of an insular pattern, with vascular invasion. (figure 2)(figure 3 )(figure 4 )

Calcitonin staining was negative, eliminating the possibility of medullary thyroid carcinoma. (calcitonin is usually expressed in medullary carcinoma and negative in follicular neoplasm).(figure 5)

Immunohistochemistry with TTF1 was positive which is mainly positive in neoplastic follicular cells. (Figure 5 )

Thyroglobulin expression is present but decreased. It was done in order to differentiate poorly differentiated carcinoma from those of medullary and anaplastic were its negative.(figure 5)

As a conclusion of the pathology, the tumor was a poorly differentiated infiltrative papillary carcinoma with an insular type and vascular invasion.

Whole body PET (positron emission tomography)-CT (computed tomography) scan performed 2 months later showed hypermetabolic foci of the right thyroid lobe with SUV (standardized uptake value) maximum of 3.7, hypermetabolic lesion in the subglottic, retrotracheal with SUV maximum of 5, concomitant with hypodense zone that could be inflammatory or metatstatic, in addition to bilateral infracentimetric suspicious lung nodules. (Figure 6 )

As a treatment, patient received 35 fractions of radiotherapy (70 Gray) planned for radioactive iodine for the remnant tissues and the metastasis.

If she failed radiotherapy and radioactive iodine, chemotherapy will be a plan as a last resort. She is well now and in a good condition.

\section{Discussion}

Thyroid cancer accounts for $1.2 \%$ among men and women as lifetime incidence, with 0.5 per 100000 per year being the estimated number of deaths.

Thyroid follicular epithelial-derived cancers are divided into three categories with papillary cancer encompassing the majority, then follicular cancer, and finally the anaplastic type. The latter, the undifferentiated type, represents less than $3 \%$ of the total affected population.

Several variants of papillary cancer have been described.

Insular variant of papillary cancer is among these forms and 

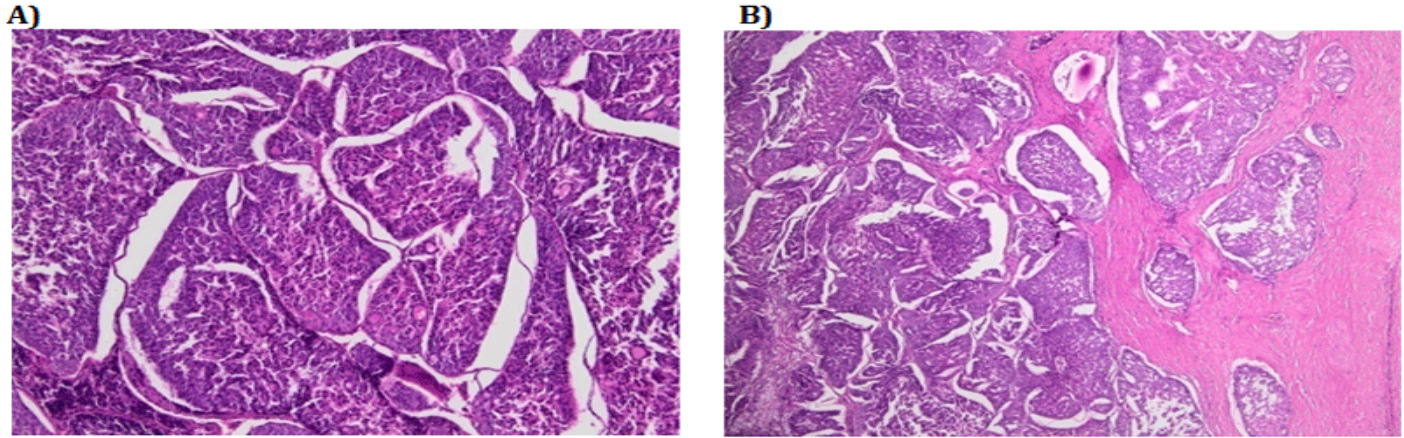

C)

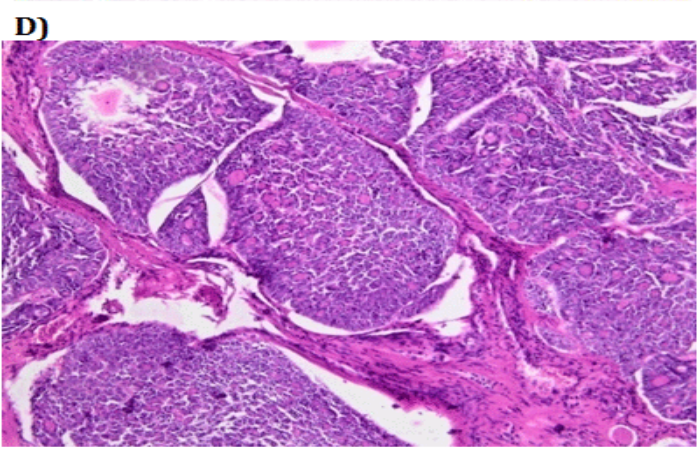

Figure 2: A) and B) Insular growth patern. C) Neoplastic follicles. D) Tumor cell nest showing artifact separation from the surrounding fibro vascular stroma.
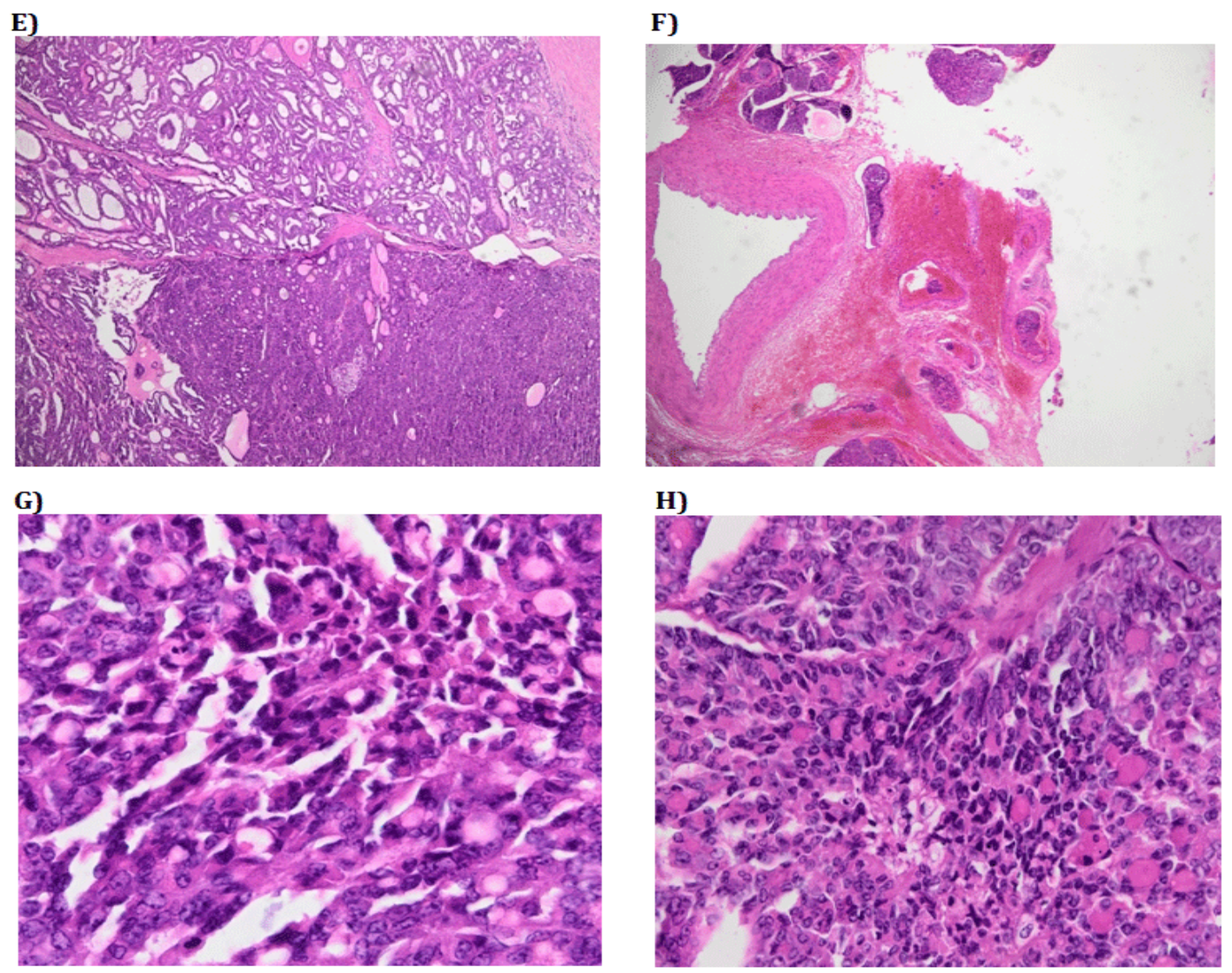

Figure 3: E) Focal presence of foci of papillary thyroid carcinoma. F) Vascular invasion. G) No nuclear features of papillary thyroid carcinoma (the nuclei are hyper chromatic and show no evidence of clearing or grooving. H) Convoluted nuclei with resinous contour. 
I)

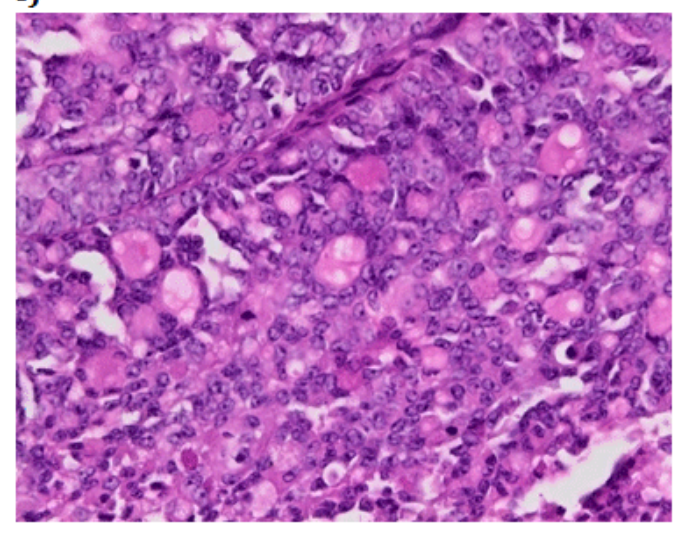

j)

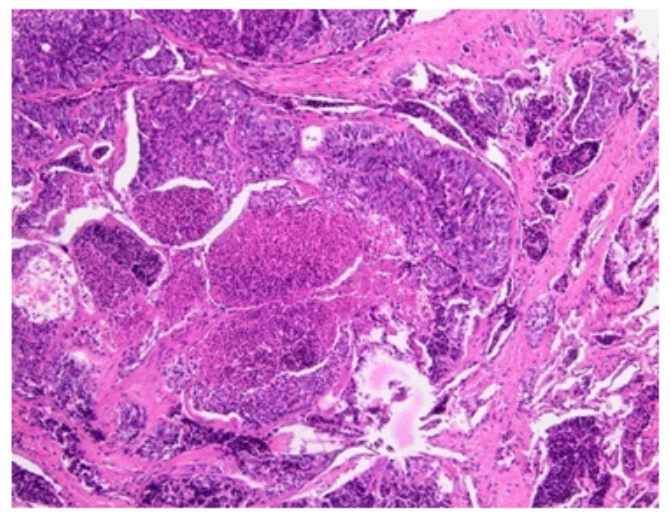

Figure 4: I) Mitosis. J) Tumor necrosis.

K)

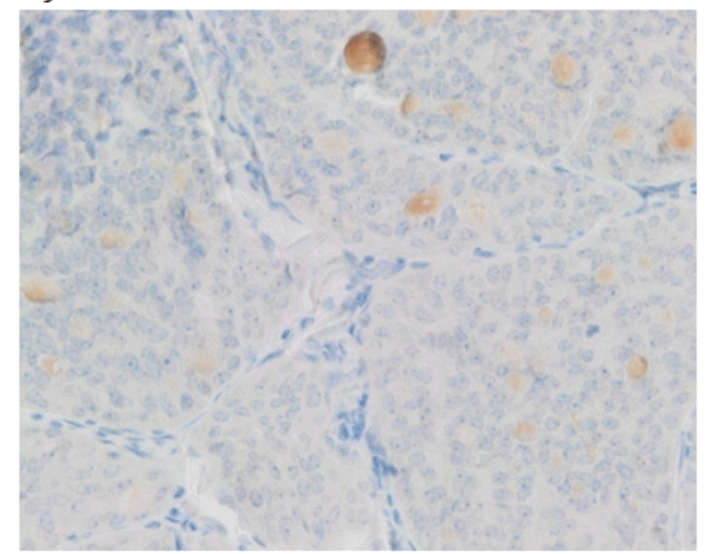

M)

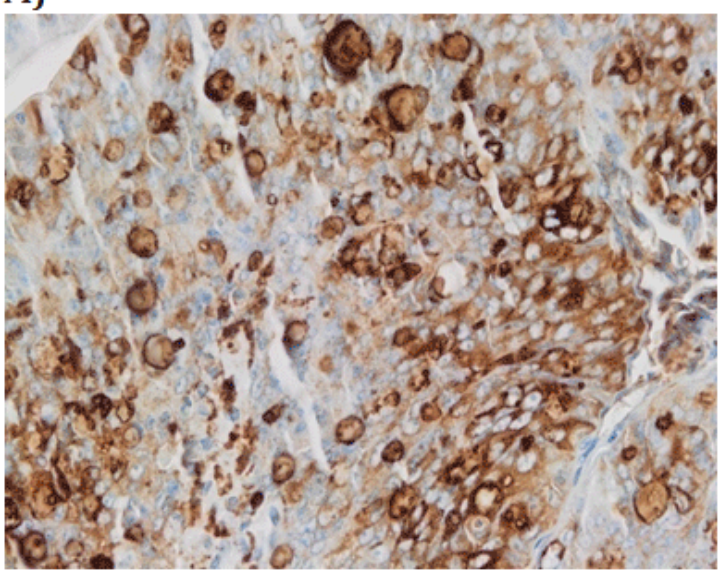

L)

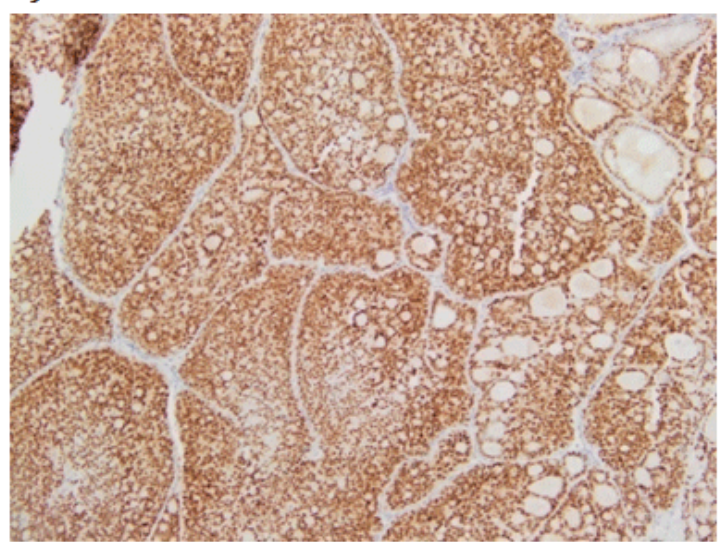

Figure 5: K) Negative calcitonin staining. L) Immunohistochemistry with TTF1 is positive. M) Thyroglobulin showed decreased expression. 


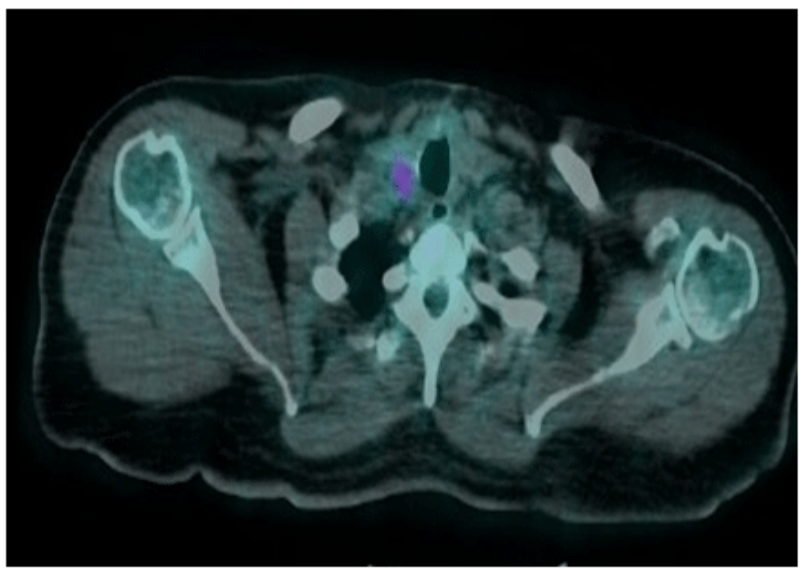

Figure 6: PET scan showing right thyroid hypermetabolic foci

is rare; with an incidence of about $5 \%$ of all thyroid tumors. All cases are reported as case reports in the literature.

Insular thyroid carcinoma was first described by carcangui et al in 1984.

It was classified in some studies as an aggressive subtype of a well differentiated cancer, and less aggressive form of a poorly differentiated thyroid cancer in other studies [2].

It occurs more commonly in females, with a median age of 55 years [3]. It carries an aggressive clinical outcome, like anaplastic thyroid cancer, with local recurrence, regional lymph node involvement and distant metastases [2].

There is a debate concerning the association between insular form of thyroid cancer and prognosis [4].

Ashfaq et al, found that this aggressive clinical feature depend on age and tumor stage rather than its insular type. However, Sasaki et al emphasized that this pattern, in addition to the other factors, are independent variables for prognosis[5]. Other studies showed that insular component does not affect prognosis and that poor survival was more related to the late disease presentation [3].

Clinical features vary; they are common to any thyroid disease and hence usually not well recognized. Calcifications with or without osseous metaplasia in the stroma occur in $23 \%$ of insular cases, which indicate a long standing disease.

$25 \%$ of patients present with long-standing goiter like our case which presented with right neck mass of long duration with calcification on thyroid ultrasound.

Diagnosis is made histologically. Islands of malignant cells are seen, small or large, round or oval, called insulae containing frequently small follicles filled by thyroglobulin. They are separated by clefts and surrounded by thin connective tissue, with capsular and vascular invasion, and necrotic foci, sometimes forming a periheliomatous pattern with variable mitotic activity [5]. Calcifications with or without osseous metaplasia in the stroma occur in $23 \%$ of insular cases, which indicate a long standing disease.
The characteristics of cells microscopically are defined as having abundant cytoplasm and rough endoplasmic reticulum, numerous mitochondria, free ribosomes, and round nuclei with chromatin dispersed homogeneously [3].

The insular thyroid cancer cytology is not well defined, even though most thyroid lesions undergo fine needle aspiration (FNA) first. In our case, FNA failed to confirm the insular variant. Studies showed that it is essential to find the exact cytological criteria preoperatively to plan an aggressive surgery once insular thyroid cancer is diagnosed. Cytologic features of poorly differentiated thyroid cancer are tall and columnar cells and others for insular type include high cellularity, low grade of atypia and clusters of neoplastic cells in microfollicules with poorly outlined cytoplasm [3].

Mutation or deletion of the p53 gene, RET, or thyroid p21 protein plays a role in thyroid cancer development, especially in anaplastic subtype [1].

Foci of insular form can be detected in anaplastic cancer. Dedifferentiation can occur and well differentiated thyroid cancer can progress to insular and later to anaplastic cancer [1].

Differential diagnoses of insular thyroid cancer include the other variants of papillary thyroid cancer, the follicular and anaplastic types [2]. As the tumor growth pattern in insular thyroid cancer is infiltrative, as proven by the case, it may be identical to that of neuroendocrine neoplasms including carcinoid or medullary thyroid cancer. Differentiation between these tumors can be identified by the presence of eosinophilic colloid-like material and thyroglobulin within the follicules of insular thyroid cancer, in addition to the absence of calcitonin, carcinoembryonic antigen and other neuroendocrine markers stain [6].

In our case, calcitonin staining was strongly negative confirming the diagnosis of insular type histologically.

Early diagnosis and close surveillance are important, because of the higher incidence of metastasis, recurrence and mortality associated with insular thyroid cancer [4]. The 5 and 10 year survival rate for patients were 46 and $42 \%$ respectively in a study with a follow up period of 10 years [1].

$20 \%$ of patients presented with distant metastases initially [3]. They were accompanied by a worse prognosis and reduced survival. (1) Bone metastases associated with poorly differentiated papillary cancer influence survival [7].

Staging of affected people is essential and is provided by AJCCTNM (The American Joint Committee on Cancer-tumor/node/ metastasis classification system) taking into consideration tumor size, lymph nodes invasion and distant metastases [4].

Total thyroidectomy and nodal resection for lymph node disease is the mainstay of treatment, even in patients with distant metastases [4]. Since this neoplasm is composed of follicular cells that concentrate iodine, this allows radioiodide imaging to detect occult metastases and to treat neoplastic deposits that may be unidentified on pre-treatment images [8]. As a result, High dose 
radioactive iodine can improve outcomes in these patients. PET scan proved metastatic disease in our patient and radioactive iodine treatment was a valuable option.

Studies of molecular changes associated with insular thyroid cancer are needed for targeted therapy and others for systemic therapy in patients with extensive disease.

\section{Conclusion}

Thyroid insular papillary carcinoma is a rare aggressive tumor, diagnosed mainly by histology, treated by thyroidectomy with lymph node resection in majority of cases, and requires early diagnosis and treatment in order to have better outcomes. It is associated with a poor prognosis especially if distant metastases are present at diagnosis.

\section{Acknowledgment}

We thank the patient for allowing us to report her case to the medical community, and for the National Institute of Pathology for providing us the pathological figures.

\section{References}

1. Lam KY, Lo CW,Chan KW, Wan KY. Insular and Anaplastic Carcinoma of the Thyroid. ANNALS OF SURGERY 2000; 231(3):329-338.

2. Salih A, Kakamad F, Aube H. Hyperfuctioning insular thyroid carcinoma: A rare case report. International Journal of Surgery Case Reports 2016;28:142-144. Doi: 10.1016/j.ijscr.2016.09.052

3. Paik SS, Kim WS,Hong EK, et al. poorly differentiated ("insular") carcinoma of the thyroid gland -two cases report. JKMS. 1997;17:7074.

4. Kazaure H, Roman S, Sosa J. Insular Thyroid Cancer. CANCER. 2012;118(13):3260-3267. Doi: 10.1002/cncr.26638

5. Albores-saavedra J, Housini I, Vuitch F, et al. Macrofollicular Variant of Papillary Thyroid Carcinoma with Minor Insular Component. CANCER 1997;80(6):1110-1116.

6. Dominguez-malagon H, Guerrero-medrano J, Suster S. Ectopic Poorly Differentiated ( Insular ) Carcinoma of the Thyroid Report of a Case Presenting as an Anterior Mediastinal Mass. AJCP. 1995;104(4):408412.

7. Tickoo S, Pittas A, Adler M, Fazzari M, Larson SM, Robbins RJ, et al. Bone Metastases From Thyroid Carcinoma A Histopathologic Study With Clinical Correlates. Arch Pathol Lab Med 2000;124(10):14401447. Doi: $10.1043 / 0003-9985(2000) 124$

8. Justin E, Seabold J, Robenson A, et al. Insular Carcinoma: A Distinct Thyroid Carcinoma with Associated Iodine- 131 Localization.The Journal of Nuclear Medicine 1991;32(7):1358-1364. 